PROCEEDINGS OF THE

AMERICAN MATHEMATICAL SOCIETY

Volume 139, Number 12, December 2011, Pages 4207-4216

S 0002-9939(2011)10889-X

Article electronically published on April 26, 2011

\title{
EMBEDDING DENDRIFORM ALGEBRA INTO ITS UNIVERSAL ENVELOPING ROTA-BAXTER ALGEBRA
}

\author{
YUQUN CHEN AND QIUHUI MO
}

(Communicated by Birge Huisgen-Zimmermann)

\begin{abstract}
In this paper, by using Gröbner-Shirshov bases for Rota-Baxter algebras, we prove that every dendriform algebra over a field of characteristic 0 can be embedded into its universal enveloping Rota-Baxter algebra.
\end{abstract}

\section{INTRODUCTION}

Let $F$ be a field. A dendriform algebra (see [10]) is an $F$-module $D$ with two binary operations $\prec$ and $\succ$ such that for any $x, y, z \in D$,

$$
\begin{aligned}
& (x \prec y) \prec z=x \prec(y \prec z+y \succ z) \\
& (x \succ y) \prec z=x \succ(y \prec z) \\
& (x \prec y+x \succ y) \succ z=x \succ(y \succ z) .
\end{aligned}
$$

Let $A$ be an associative algebra over $F$. Let an $F$-linear operator $P: A \rightarrow A$ satisfy the Rota-Baxter identity

$$
P(x) P(y)=P(P(x) y)+P(x P(y)) .
$$

Then $A$ is called a Rota-Baxter algebra.

The free Rota-Baxter algebra generated by a nonempty set $X$, denoted by $R B(X)$, was given by K. Ebrahimi-Fard and L. Guo [7] and the free dendriform algebra generated by $X$, denoted by $D(X)$, was first made explicit by J.-L. Loday in [10].

Suppose that $(D, \prec, \succ)$ is a dendriform algebra over $F$ with a linear basis $X=$ $\left\{x_{i} \mid i \in I\right\}$. Let $x_{i} \prec x_{j}=\left\{x_{i} \prec x_{j}\right\}, x_{i} \succ x_{j}=\left\{x_{i} \succ x_{j}\right\}$, where $\left\{x_{i} \prec x_{j}\right\}$ and $\left\{x_{i} \succ x_{j}\right\}$ are linear combinations of $x \in X$. Then $D$ has an expression by generators and defining relations

$$
D=D\left(X \mid x_{i} \prec x_{j}=\left\{x_{i} \prec x_{j}\right\}, x_{i} \succ x_{j}=\left\{x_{i} \succ x_{j}\right\}, x_{i}, x_{j} \in X\right) .
$$

Denote by

$$
U(D)=R B\left(X \mid x_{i} P\left(x_{j}\right)=\left\{x_{i} \prec x_{j}\right\}, P\left(x_{i}\right) x_{j}=\left\{x_{i} \succ x_{j}\right\}, x_{i}, x_{j} \in X\right) .
$$

Received by the editors April 20, 2010 and, in revised form, August 27, 2010 and October 20, 2010 .

2010 Mathematics Subject Classification. Primary 13P10, 16S15; Secondary 16W99, 17A50.

Key words and phrases. Gröbner-Shirshov basis, universal enveloping algebra, dendriform algebra, Rota-Baxter algebra.

The first author was supported in part by the NNSF of China (Nos. 10771077, 10911120389) and the NSF of Guangdong Province (No. 06025062). 
Then $U(D)$ is the universal enveloping Rota-Baxter algebra of $D$; see [7.

The study of Rota-Baxter algebras originated from the probability study of Glenn Baxter in 1960 and was developed further by Cartier and the school of Rota in the 1960s and 1970s. This structure appeared also in the Lie algebra context as the operator form of the classical Yang-Baxter equation started in the 1980s. Since then, Rota-Baxter algebra has experienced a quite remarkable renaissance and found important theoretical developments and applications in mathematical physics, operads, number theory and combinatorics; see, for example, [1, 3, 5, 6, 8, 13.

The dendriform algebra was introduced by J.-L. Loday [10] in 1995 with motivation from algebraic K-theory, and was further studied in connection with several areas in mathematics and physics, including operads, homology, Hopf algebras, Lie and Leibniz algebras, combinatorics, arithmetic and quantum field theory; see 7, 11.

In the theory of Lie algebras, the Poincaré-Birkhoff-Witt theorem (see [12, frequently contracted to the PBW theorem) is a fundamental result giving an explicit description of the universal enveloping algebra of a Lie algebra. The term "PBW type theorem" or even "PBW theorem" may also refer to various analogues of the original theorem. I. P. Shestakov [14 proved that an Akivis algebra can be embedded into its universal enveloping non-associative algebra. M. Aymon and P.-P. Grivel [2] proved that a Leibniz algebra can be embedded into its universal enveloping diassociative algebra. P. S. Kolesnikov 9] proved that every (finite dimensional) Leibniz algebra can be embedded into current conformal algebra over the algebra of linear transformations of a (finite dimensional) linear space. As a corollary, a new proof of the theorem on injective embeddings of a Leibniz algebra into an diassociative algebra is obtained and, more explicitly, an analogue of the PBW theorem for Leibniz algebras in [10].

In this paper we study the functor from Rota-Baxter algebras to dendriform algebras given by the formulas $x \prec y:=x P(y), x \succ y:=P(x) y$. The identities defining a dendriform algebra are a consequence of the associativity of the product in a Rota-Baxter algebra and of the Rota-Baxter identity $P(x) P(y)=x P(y)+$ $P(x) y$, which reads $P(x) P(y)=P(x \prec y+x \succ y)$. This functor is a forgetful functor; hence it admits a left adjoint, denoted by $U$, and given, as usual, by the quotient of the free Rota-Baxter algebra by the relations which identify the two dendriform structures. From the universal property of the enveloping functor $U$ it comes immediately that there is a natural map $D \rightarrow U(D)$ for any dendriform algebra $D$. It is the unit of the adjunction. Similar to a classical problem involving associative and Lie algebras, L. Guo posts the following conjecture: each dendriform algebra can be embedded into its universal enveloping Rota-Baxter algebra; i.e., the map $D \rightarrow U(D)$ is injective.

In this paper, we prove the following theorem.

Theorem 1.1. Every dendriform algebra over a field of characteristic 0 can be embedded into its universal enveloping Rota-Baxter algebra. In other words, such a dendriform algebra is isomorphic to a dendriform subalgebra of a Rota-Baxter algebra.

The Composition-Diamond lemma for Rota-Baxter algebras was established by L. A. Bokut, Yuqun Chen and Xueming Deng in a recent paper 4. We will use this lemma to prove the above theorem. 


\section{Preliminaries}

In this section, we introduce some notations which are related to GröbnerShirshov bases for Rota-Baxter algebras; see [4].

Let $X$ be a nonempty set, $S(X)$ the free semigroup generated by $X$ without identity and $P$ a symbol of a unary operation. For any two nonempty sets $Y$ and $Z$, denote by

$$
\begin{aligned}
\Lambda_{P}(Y, Z)= & \left(\bigcup_{r \geq 0}(Y P(Z))^{r} Y\right) \cup\left(\bigcup_{r \geq 1}(Y P(Z))^{r}\right) \\
& \cup\left(\bigcup_{r \geq 0}(P(Z) Y)^{r} P(Z)\right) \cup\left(\bigcup_{r \geq 1}(P(Z) Y)^{r}\right),
\end{aligned}
$$

where for a set $T, T^{r}=\left\{t_{1} \cdots t_{r} \mid t_{i} \in T, 1 \leq i \leq r\right\}$ and $T^{0}$ means the empty set.

Define

$$
\begin{array}{ccc}
\Phi_{0} & = & S(X) \\
\vdots & & \vdots \\
\Phi_{n} & = & \Lambda_{P}\left(\Phi_{0}, \Phi_{n-1}\right) \\
\vdots & & \vdots
\end{array}
$$

Then

$$
\Phi_{0} \subset \cdots \subset \Phi_{n} \subset \cdots
$$

Let

$$
\Phi(X)=\bigcup_{n \geq 0} \Phi_{n}
$$

Clearly, $P(\Phi(X)) \subset \Phi(X)$. If $u \in X \cup P(\Phi(X))$, then $u$ is called prime. For any $u \in \Phi(X), u$ has a unique form $u=u_{1} u_{2} \cdots u_{n}$ where $u_{i}$ is prime, $i=1,2, \ldots, n$, and $u_{i}, u_{i+1}$ cannot both have forms as $P\left(u_{i}^{\prime}\right)$ and $P\left(u_{i+1}^{\prime}\right)$.

For any $u \in \Phi(X)$ and for a set $T \subseteq X \cup\{P\}$, denote by $\operatorname{deg}_{T}(u)$ the number of occurrences of $t \in T$ in $u$. Let

$$
\operatorname{Deg}(u)=\left(\operatorname{deg}_{\{P\} \cup X}(u), \operatorname{deg}_{\{P\}}(u)\right) .
$$

We order $\operatorname{Deg}(u)$ lexicographically.

In the following, we always assume that $F$ is a field of characteristic 0 .

Let $F \Phi(X)$ be a free $F$-module with $F$-basis $\Phi(X)$. Extend linearly

$$
P: F \Phi(X) \rightarrow F \Phi(X), u \mapsto P(u),
$$

where $u \in \Phi(X)$.

Now we define the multiplication in $F \Phi(X)$.

Firstly, for $u, v \in X \cup P(\Phi(X))$, define

$$
u \cdot v= \begin{cases}P\left(P\left(u^{\prime}\right) \cdot v^{\prime}\right)+P\left(u^{\prime} \cdot P\left(v^{\prime}\right)\right), & \text { if } u=P\left(u^{\prime}\right), v=P\left(v^{\prime}\right) ; \\ u v, & \text { otherwise. }\end{cases}
$$

Secondly, for any $u=u_{1} u_{2} \cdots u_{s}, v=v_{1} v_{2} \cdots v_{l} \in \Phi(X)$, where $u_{i}, v_{j}$ are prime, $i=1,2, \ldots, s, j=1,2, \ldots, l$, define

$$
u \cdot v=u_{1} u_{2} \cdots u_{s-1}\left(u_{s} \cdot v_{1}\right) v_{2} \cdots v_{l} .
$$


Equipped with the above concepts, $F \Phi(X)$ is the free Rota-Baxter algebra generated by $X$; see 7 .

We denote by $R B(X)$ the free Rota-Baxter algebra generated by $X$.

Let $N^{+}$be the set of positive integers.

Let the notation be as before. We have to order $\Phi(X)$. Let $X$ be a well-ordered set. Let us define an ordering $>$ on $\Phi(X)$ by induction on the $D e g$-function.

For any $u, v \in \Phi(X)$, if $\operatorname{Deg}(u)>\operatorname{Deg}(v)$, then $u>v$. If $\operatorname{Deg}(u)=\operatorname{Deg}(v)=$ $(n, m)$, then we define $u>v$ by induction on $(n, m)$.

If $(n, m)=(1,0)$, then $u, v \in X$ and we use the ordering on $X$. Suppose that for $(n, m)$ the ordering is defined where $(n, m) \geq(1,0)$. Let $(n, m)<\left(n^{\prime}, m^{\prime}\right)=$ $\operatorname{Deg}(u)=\operatorname{Deg}(v)$. If $u, v \in P(\Phi(X))$, say $u=P\left(u^{\prime}\right)$ and $v=P\left(v^{\prime}\right)$, then $u>v$ if and only if $u^{\prime}>v^{\prime}$ by induction. Otherwise $u=u_{1} u_{2} \cdots u_{l}$ and $v=v_{1} v_{2} \cdots v_{s}$ where $l>1$ or $s>1$, and $u>v$ if and only if $\left(u_{1}, u_{2}, \ldots, u_{l}\right)>\left(v_{1}, v_{2}, \ldots, v_{s}\right)$ lexicographically by induction.

It is clear that $>$ is a well ordering on $\Phi(X)$; see [4]. Throughout this paper, we will use this ordering.

Let $\star$ be a symbol and $\star \notin X$. By a $\star$-Rota-Baxter word we mean any expression in $\Phi(X \cup\{\star\})$ with only one occurrence of $\star$. The set of all $\star$-Rota-Baxter words on $X$ is denoted by $\Phi^{\star}(X)$.

Let $u$ be a $\star$-Rota-Baxter word and $s \in R B(X)$. Then we call

$$
\left.u\right|_{s}=\left.u\right|_{\star \mapsto s}
$$

an $s$-Rota-Baxter word. For short, we call $\left.u\right|_{s}$ an $s$-word.

Note that the ordering $>$ is monomial in the sense that for any $u, v \in \Phi(X), w \in$ $\Phi^{\star}(X)$

$$
u>v \Longrightarrow \overline{\left.w\right|_{u}}>\overline{\left.w\right|_{v}},
$$

where $\left.w\right|_{u}=\left.w\right|_{\star \mapsto u}$ and $\left.w\right|_{v}=\left.w\right|_{\star \mapsto v}$; see [4], Lemma 3.4.

If $\overline{\left.u\right|_{s}}=\left.u\right|_{\bar{s}}$, then we call $\left.u\right|_{s}$ a normal $s$-word.

Now, for any $0 \neq f \in R B(X), f$ has the leading term $\bar{f}$ and $f=\alpha_{1} \bar{f}+\sum_{i=2}^{n} \alpha_{i} u_{i}$, where $\bar{f}, u_{i} \in \Phi(X), \bar{f}>u_{i}, 0 \neq \alpha_{1}, \alpha_{i} \in F$. Denote by $l c(f)$ the coefficient of the leading term $\bar{f}$. If $l c(f)=1$, then we call $f$ monic.

Let $f, g \in R B(X)$ be monic with $\bar{f}=u_{1} u_{2} \cdots u_{n}$ where each $u_{i}$ is prime. Then, there are four kinds of compositions:

(1) If $u_{n} \in P(\Phi(X))$, then we define the composition of right multiplication as $f \cdot u$, where $u \in P(\Phi(X))$.

(2) If $u_{1} \in P(\Phi(X))$, then we define the composition of left multiplication as $u \cdot f$, where $u \in P(\Phi(X))$.

(3) If there exists a $w=\bar{f} a=b \bar{g}$ where $f a$ is a normal $f$-word and $b g$ is a normal $g$-word, $a, b \in \Phi(X)$ and $\operatorname{deg}_{\{P\} \cup X}(w)<\operatorname{deg}_{\{P\} \cup X}(\bar{f})+\operatorname{deg}_{\{P\} \cup X}(\bar{g})$, then we define the intersection composition of $f$ and $g$ with respect to $w$ as $(f, g)_{w}=f \cdot a-b \cdot g$.

(4) If there exists a $w=\bar{f}=\left.u\right|_{\bar{g}}$ where $u \in \Phi^{\star}(X)$, then we define the inclusion composition of $f$ and $g$ with respect to $w$ as $(f, g)_{w}=f-\left.u\right|_{g}$.

We call $w$ in $(f, g)_{w}$ the ambiguity with respect to $f$ and $g$.

Let $S \subset R B(X)$ be a set of monic polynomials. Then the composition $h$ is called trivial modulo $(S, w)$, denoted by $h \equiv 0 \bmod (S, w)$, if

$$
h=\left.\sum_{i} \alpha_{i} u_{i}\right|_{s_{i}}
$$


where each $\alpha_{i} \in F, s_{i} \in S,\left.u_{i}\right|_{s_{i}}$ is a normal $s_{i}$-word and $\left.u_{i}\right|_{\overline{s_{i}}}<w\left(\left.u_{i}\right|_{\overline{s_{i}}} \leq \bar{h}\right.$ if $h$ is a composition of left (right) multiplication).

In general, for any two polynomials $p$ and $q, p \equiv q \bmod (S, w)$ means that $p-q \equiv$ $0 \bmod (S, w)$.

The set $S$ is called a Gröbner-Shirshov basis in $R B(X)$ if each composition is trivial modulo $S$ and corresponding $w$.

Theorem 2.1 (Composition-Diamond lemma for Rota-Baxter algebras [4]). Let $R B(X)$ be a free Rota-Baxter algebra over a field of characteristic 0 and let $S$ be a set of monic polynomials in $R B(X),>$ the monomial ordering on $\Phi(X)$ defined as before and $\operatorname{Id}(S)$ the Rota-Baxter ideal of $R B(X)$ generated by $S$. Then the following statements are equivalent:

(1) $S$ is a Gröbner-Shirshov basis in $R B(X)$.

(2) $f \in I d(S) \Rightarrow \bar{f}=\left.u\right|_{\bar{s}}$ for some $u \in \Phi^{\star}(X), s \in S$.

(3) $\operatorname{Irr}(S)=\left\{u \in \Phi(X)|u \neq v|_{\bar{s}}, s \in S,\left.v\right|_{s}\right.$ is a normal s-word $\}$ is an F-basis of $R B(X \mid S)=R B(X) / I d(S)$.

If a subset $S$ of $R B(X)$ is not a Gröbner-Shirshov basis, then one can add all nontrivial compositions of polynomials of $S$ to $S$. Continuing this process repeatedly, we finally obtain a Gröbner-Shirshov basis $S^{\text {comp }}$ that contains $S$. Such a process is called the Shirshov algorithm.

\section{The Proof of Theorem 1.1}

In this section, we assume that $R B(X)$ is the free Rota-Baxter algebra generated by $X=\left\{x_{i} \mid i \in I\right\}$.

Lemma 3.1. For any $u, v \in \Phi(X)$, we have $\overline{P(u) P(v)}=\max \{\overline{P(P(u) v)}, \overline{P(u P(v))}\}$.

Proof. By Rota-Baxter formula (1.2), we may assume that $P(P(u) v)=\sum n_{i} u_{i}$, $P(u P(v))=\sum m_{j} v_{j}$, where $n_{i}, m_{j} \in N^{+}, u_{i}, v_{j} \in \Phi(X)$. Since the characteristic of $F$ is 0 , the result follows.

Denote by

$$
\begin{aligned}
& F_{1}=\left\{x_{i} P\left(x_{j}\right)-\left\{x_{i} \prec x_{j}\right\} \mid i, j \in I\right\}, \\
& F_{2}=\left\{P\left(x_{i}\right) x_{j}-\left\{x_{i} \succ x_{j}\right\} \mid i, j \in I\right\}, \\
& \operatorname{Irr}\left(F_{1} \cup F_{2}\right)=\left\{u \in \Phi(X)|u \neq v|_{\bar{s}}, s \in F_{1} \cup F_{2},\left.v\right|_{s} \text { is a normal } s \text {-word }\right\}, \\
& \Phi_{1}(X)=\Phi(X) \cap \operatorname{Irr}\left(F_{1} \cup F_{2}\right) .
\end{aligned}
$$

For a polynomial $f=\sum_{i=1}^{n} \alpha_{i} u_{i} \in R B(X)$, where each $0 \neq \alpha_{i} \in F, u_{i} \in \Phi(X)$, denote the set $\left\{u_{i}, 1 \leq i \leq n\right\}$ by $\operatorname{supp}(f)$.

Lemma 3.2. 1) Let $f=P\left(x_{i}\right) u, g=v P\left(x_{j}\right)$, where $i, j \in I, u, v \in \Phi_{1}(X) \backslash X$. Then $f \equiv \sum \alpha_{i} u_{i} \bmod \left(F_{1} \cup F_{2}, \bar{f}\right)$ and $g \equiv \sum \beta_{i} v_{i} \bmod \left(F_{1} \cup F_{2}, \bar{g}\right)$, where for any $i, \alpha_{i}, \beta_{i} \in F, u_{i}, v_{i} \in \Phi_{1}(X) \backslash X$.

2) Let $f=P(u) P(v), g=P\left(v^{\prime}\right) P\left(u^{\prime}\right)$, where $u, u^{\prime} \in \Phi_{1}(X) \backslash X, v, v^{\prime} \in \Phi_{1}(X)$. Then $f \equiv \sum \alpha_{i} P\left(u_{i}\right) \bmod \left(F_{1} \cup F_{2}, \bar{f}\right)$ and $g \equiv \sum \beta_{i} P\left(v_{i}\right) \bmod \left(F_{1} \cup F_{2}, \bar{g}\right)$, where for any $i, \alpha_{i}, \beta_{i} \in F, u_{i}, v_{i} \in \Phi_{1}(X) \backslash X$.

Proof. 1) We prove only the case when $f=P\left(x_{i}\right) u \equiv \sum \alpha_{i} u_{i} \bmod \left(F_{1} \cup F_{2}, \bar{f}\right)$. The other case is similar.

We use induction on $n=\operatorname{deg}_{\{P\} \cup X}(u)$. Since $u \in \Phi_{1}(X) \backslash X$, we have $n \geq 2$. 
Assume that $n=2$. Then either $u=x_{j} x_{k}$ or $u=P(x), x_{j}, x_{k}, x \in X$. If $u=x_{j} x_{k}$, then we have $f=P\left(x_{i}\right) x_{j} x_{k} \equiv\left\{x_{i} \succ x_{j}\right\} x_{k} \bmod \left(F_{1} \cup F_{2}, \bar{f}\right)$, and $\operatorname{supp}\left(\left\{x_{i} \succ x_{j}\right\} x_{k}\right) \subset \Phi_{1}(X) \backslash X$. If $u=P(x)$, then $f=P\left(x_{i}\right) P(x)=$ $P\left(P\left(x_{i}\right) x\right)+P\left(x_{i} P(x)\right) \equiv P\left(\left\{x_{i} \succ x\right\}\right)+P\left(\left\{x_{i} \prec x\right\}\right) \bmod \left(F_{1} \cup F_{2}, \bar{f}\right)$, and $\operatorname{supp}\left(P\left(\left\{x_{i} \succ x\right\}\right)\right), \operatorname{supp}\left(P\left(\left\{x_{i} \prec x\right\}\right)\right) \subset \Phi_{1}(X) \backslash X$.

For $n>2$, there are three cases to consider.

(1) $u=x_{j} u_{1}, x_{j} \in X$. Then there are two subcases to consider.

(a) $u_{1}=x_{k} u_{2}, x_{k} \in X$. Then $f=P\left(x_{i}\right) u \equiv\left\{x_{i} \succ x_{j}\right\} x_{k} u_{2} \quad \bmod \left(F_{1} \cup\right.$ $\left.F_{2}, \bar{f}\right)$, where $\operatorname{supp}\left(\left\{x_{i} \succ x_{j}\right\} x_{k} u_{2}\right) \subset \Phi_{1}(X) \backslash X$.

(b) $u_{1}=P(v) u_{2}$. Since $u \in \Phi_{1}(X) \backslash X$, we get that $v \notin X$. Thus, $f=P\left(x_{i}\right) u \equiv\left\{x_{i} \succ x_{j}\right\} P(v) u_{2} \bmod \left(F_{1} \cup F_{2}, \bar{f}\right)$, where $\operatorname{supp}\left(\left\{x_{i} \succ x_{j}\right\} P(v) u_{2}\right) \subset \Phi_{1}(X) \backslash X$.

(2) $u=P\left(u_{1}\right)$. Then

$$
f=P\left(x_{i}\right) u=P\left(x_{i}\right) P\left(u_{1}\right)=P\left(P\left(x_{i}\right) u_{1}\right)+P\left(x_{i} P\left(u_{1}\right)\right) .
$$

Let

and

$$
P\left(x_{i}\right) u_{1} \equiv \sum \gamma_{i} w_{i} \bmod \left(F_{1} \cup F_{2}, \overline{P\left(x_{i}\right) u_{1}}\right)
$$

$$
x_{i} P\left(u_{1}\right) \equiv \sum \gamma_{i}^{\prime} w_{i}^{\prime} \bmod \left(F_{1} \cup F_{2}, \overline{x_{i} P\left(u_{1}\right)}\right),
$$

where all $w_{i}, w_{i}^{\prime} \in \Phi_{1}(X)$. By using Lemma 3.1, $\bar{f}=\overline{P\left(x_{i}\right) P\left(u_{1}\right)} \geq$ $\overline{P\left(P\left(x_{i}\right) u_{1}\right)}=P\left(\overline{P\left(x_{i}\right) u_{1}}\right) \geq P\left(w_{j}\right)$ and similarly, $\bar{f} \geq P\left(w_{j}^{\prime}\right)$ for any $j, j^{\prime}$. Then $f \equiv \sum \gamma_{i} P\left(w_{i}\right)+\sum \gamma_{i}^{\prime} P\left(w_{i}^{\prime}\right) \bmod \left(F_{1} \cup F_{2}, \bar{f}\right)$, where $P\left(w_{i}\right), P\left(w_{i}^{\prime}\right) \in$ $\Phi_{1}(X) \backslash X$.

(3) $u=P\left(u_{1}\right) u_{2}$, where $u_{2}$ is not empty. Then $u_{1} \notin X$, and $u_{2}=x_{j} u_{3}$ for some $x_{j} \in X$ since $u \in \Phi_{1}(X) \backslash X$. Therefore, $f=P\left(x_{i}\right) u=P\left(x_{i}\right) P\left(u_{1}\right) u_{2}=$ $P\left(P\left(x_{i}\right) u_{1}\right) u_{2}+P\left(x_{i} P\left(u_{1}\right)\right) u_{2}$. For $P\left(x_{i} P\left(u_{1}\right)\right) u_{2}$, we have $P\left(x_{i} P\left(u_{1}\right)\right) u_{2} \in$ $\Phi_{1}(X) \backslash X$. For $P\left(P\left(x_{i}\right) u_{1}\right) u_{2}$, since $u_{1} \notin X$, by induction on $n$, we get that $P\left(x_{i}\right) u_{1} \equiv \sum \gamma_{i} v_{i} \bmod \left(F_{1} \cup F_{2}, \overline{P\left(x_{i}\right) u_{1}}\right)$, where $v_{i} \in \Phi_{1}(X) \backslash X$. By using Lemma 3.1, $\bar{f}=\overline{P\left(x_{i}\right) P\left(u_{1}\right) u_{2}} \geq \overline{P\left(P\left(x_{i}\right) u_{1}\right) u_{2}}=P\left(\overline{P\left(x_{i}\right) u_{1}}\right) u_{2} \geq$ $P\left(v_{i}\right) u_{2}$. As a result, $P\left(P\left(x_{i}\right) u_{1}\right) u_{2} \equiv \sum \gamma_{i} P\left(v_{i}\right) u_{2} \bmod \left(F_{1} \cup F_{2}, \bar{f}\right)$ and $P\left(v_{i}\right) u_{2} \in \Phi_{1}(X) \backslash X$.

2) We only prove the case $f=P(u) P(v)$. The other case is proved similarly.

We use induction on $n=\operatorname{deg}_{\{P\} \cup X}(P(u) P(v))$. Since $u \in \Phi_{1}(X) \backslash X$, we have $n \geq 5$.

Assume that $n=5$. Then either $u=x_{i} x_{j}$ and $v=x$ or $u=P\left(x_{i}\right)$ and $v=x$, where $x_{i}, x_{j}, x \in X$.

If $u=x_{i} x_{j}$ and $v=x$, then we have $f=P(u) P(v)=P\left(x_{i} x_{j}\right) P(x)=$ $P\left(P\left(x_{i} x_{j}\right) x\right)+P\left(x_{i} x_{j} P(x)\right) \equiv P\left(P\left(x_{i} x_{j}\right) x\right)+P\left(x_{i}\left\{x_{j} \prec x\right\}\right) \bmod \left(F_{1} \cup F_{2}, \bar{f}\right)$, and $\left(\left\{P\left(x_{i} x_{j}\right) x\right\} \cup \operatorname{supp}\left(x_{i}\left\{x_{j} \prec x\right\}\right)\right) \subset \Phi_{1}(X) \backslash X$.

If $u=P\left(x_{i}\right)$ and $v=x$, then $f=P(u) P(v)=P\left(P\left(x_{i}\right)\right) P(x)=P\left(P\left(P\left(x_{i}\right)\right) x\right)+$ $P\left(P\left(x_{i}\right) P(x)\right)=P\left(P\left(P\left(x_{i}\right)\right) x\right)+P\left(P\left(P\left(x_{i}\right) x\right)\right)+P\left(P\left(x_{i} P(x)\right)\right) \equiv P\left(P\left(P\left(x_{i}\right)\right) x\right)+$ $P\left(P\left(\left\{x_{i} \succ x\right\}\right)\right)+P\left(P\left(\left\{x_{i} \prec x\right\}\right)\right) \bmod \left(F_{1} \cup F_{2}, \bar{f}\right)$, and $\left(\left\{P\left(P\left(x_{i}\right)\right) x\right\} \cup\right.$ $\left.\operatorname{supp}\left(P\left(\left\{x_{i} \succ x\right\}\right)\right) \cup \operatorname{supp}\left(P\left(\left\{x_{i} \prec x\right\}\right)\right)\right) \subset \Phi_{1}(X) \backslash X$.

For $n>5$, since $f=P(u) P(v)=P(u P(v))+P(P(u) v)$ and by Lemma 3.1. it is sufficient to prove that $P(u P(v)) \equiv \sum \alpha_{i} P\left(u_{i}\right) \bmod \left(F_{1} \cup F_{2}, \overline{P(u P(v))}\right)$, $P(P(u) v) \equiv \sum \alpha_{i} P\left(v_{i}\right) \bmod \left(F_{1} \cup F_{2}, \overline{P(P(u) v)}\right)$, where $u_{i}, v_{i} \in \Phi_{1}(X) \backslash X$.

For $P(u P(v))$, there are two cases to consider. 
(1) $u=u_{1} x_{i}, x_{i} \in X$. Then there are two subcases to consider.

(a) $v \notin X$. Then $P(u P(v))=P\left(u_{1} x_{i} P(v)\right)$ and $u_{1} x_{i} P(v) \in \Phi_{1}(X) \backslash X$.

(b) $v=x_{j} \in X$. Then $P(u P(v))=P\left(u_{1} x_{i} P\left(x_{j}\right)\right) \equiv P\left(u_{1}\left\{x_{i} \prec x_{j}\right\}\right)$. If $u_{1}=u_{2} x$ for some $x \in X$, then $P(u P(v)) \equiv P\left(u_{2} x\left\{x_{i} \prec x_{j}\right\}\right)$ where $\operatorname{supp}\left(u_{2} x\left\{x_{i} \prec x_{j}\right\}\right) \subset \Phi_{1}(X) \backslash X$. If $u_{1}=u_{2} P\left(u_{3}\right)$, then $u=$ $u_{1} x_{i}=u_{2} P\left(u_{3}\right) x_{i}$ and $u_{3} \notin X$. Then $P(u P(v)) \equiv P\left(u_{1}\left\{x_{i} \prec x_{j}\right\}\right) \equiv$ $P\left(u_{2} P\left(u_{3}\right)\left\{x_{i} \prec x_{j}\right\}\right)$ where $\operatorname{supp}\left(u_{2} P\left(u_{3}\right)\left\{x_{i} \prec x_{j}\right\}\right) \subset \Phi_{1}(X) \backslash X$.

(2) $u=u_{1} P\left(u_{2}\right)$. Then there are two subcases to consider.

(a) $u_{2}=x_{i} \in X$. Since $u \in \Phi_{1}(X) \backslash X$, we have $u=P\left(x_{i}\right)$. As a result, $P(u P(v))=P\left(P\left(x_{i}\right) P(v)\right)$. Since $P(v) \notin X$, the result follows from $1)$.

(b) $u_{2} \notin X$. Then $u_{2} \in \Phi_{1}(X) \backslash X$ and $P(u P(v))=P\left(u_{1} P\left(u_{2}\right) P(v)\right)$. By induction on $n, P\left(u_{2}\right) P(v) \equiv \sum \alpha_{i} P\left(v_{i}\right) \bmod \left(F_{1} \cup F_{2}, \overline{P\left(u_{2}\right) P(v)}\right)$, where $v_{i} \in \Phi_{1}(X) \backslash X$. Then $P(u P(v))=P\left(u_{1} P\left(u_{2}\right) P(v)\right) \equiv$ $\sum \alpha_{i} P\left(u_{1} P\left(v_{i}\right)\right)$ and $u_{1} P\left(v_{i}\right) \in \Phi_{1}(X) \backslash X$.

For $P(P(u) v)$, there are also two cases to consider.

(1) $v=x_{i} v_{1}, x_{i} \in X$. Then $P(P(u) v)=P\left(P(u) x_{i} v_{1}\right)$ and $P(u) x_{i} v_{1} \in$ $\Phi_{1}(X) \backslash X$.

(2) $v=P\left(v_{1}\right) v_{2}$. Then $P(P(u) v)=P\left(P(u) P\left(v_{1}\right) v_{2}\right)$ with $v_{1} \in \Phi_{1}(X)$. By induction on $n$, we get that $P(u) P\left(v_{1}\right) \equiv \sum \alpha_{i} P\left(u_{i}\right) \bmod \left(F_{1} \cup F_{2}, \overline{P(u) P\left(v_{1}\right)}\right)$, where $u_{i} \in \Phi_{1}(X) \backslash X$. Then $P(P(u) v) \equiv \sum \alpha_{i} P\left(P\left(u_{i}\right) v_{2}\right)$ and $P\left(u_{i}\right) v_{2} \in$ $\Phi_{1}(X) \backslash X$.

The proof is complete.

Lemma 3.3. Let $S=F_{1} \cup F_{2} \cup F_{3}$, where

$$
\begin{aligned}
F_{3}= & \left\{u_{0} P\left(v_{1}\right) u_{1} \cdots P\left(v_{n}\right) u_{n} \mid u_{0}, u_{n} \in X^{*}, u_{i} \in X^{*} \backslash\{1\}, 1 \leq i<n,\right. \\
& \left.v_{j} \in \Phi_{1}(X) \backslash X, 1 \leq j \leq n ;\left|u_{0}\right| \geq 2 \text { if } n=0\right\}
\end{aligned}
$$

for any $u \in X^{*},|u|$ is the length of $u$, and $X^{*}$ is the free monoid generated by $X$.

Then $S$ is a Gröbner-Shirshov basis in $R B(X)$.

Proof. The ambiguities of all possible compositions of the polynomials in $S$ are listed below:

$f_{1} \wedge f_{2}: \quad f_{1} \in F_{1}, f_{2} \in F_{2}$, and $w=x_{i} P\left(x_{j}\right) x_{k}, i, j, k \in I$.

$f_{2} \wedge f_{1}: \quad f_{1} \in F_{1}, f_{2} \in F_{2}$, and $w=P\left(x_{i}\right) x_{j} P\left(x_{k}\right), i, j, k \in I$.

$f_{2} \wedge f_{3}: \quad f_{2} \in F_{2}, f_{3} \in F_{3}$, and $w=P\left(x_{i}\right) x_{j} u_{0} P\left(v_{1}\right) u_{1} \cdots P\left(v_{n}\right) u_{n}, u_{0}, u_{n} \in$ $X^{*}, u_{k} \in X^{*} \backslash\{1\}, v_{l} \in \Phi_{1}(X) \backslash X, i, j \in I, n \geq 0,1 \leq k<n, 1 \leq l \leq n$. When $n=0,\left|u_{0}^{(1)}\right| \geq 1$.

$f_{3} \wedge f_{1}: \quad f_{1} \in F_{1}, f_{3} \in F_{3}$, and $w=u_{0} P\left(v_{1}\right) u_{1} \cdots P\left(v_{n}\right) u_{n} x_{i} P\left(x_{j}\right), u_{0}, u_{n} \in$ $X^{*}, u_{k} \in X^{*} \backslash\{1\}, v_{l} \in \Phi_{1}(X) \backslash X, i, j \in I, n \geq 0,1 \leq k<n, 1 \leq l \leq n$. When $n=0,\left|u_{0}^{(1)}\right| \geq 1$.

$f_{3} \wedge f_{3}^{\prime}: \quad f_{3}, f_{3}^{\prime} \in F_{3}$. There are three ambiguities: one is for the intersection composition, and two are for the inclusion composition.

All possible compositions of left and right multiplication are: $f_{1} P(u), P(u) f_{2}$, $f_{3} P(u)$ and $P(u) f_{3}$, where $f_{i} \in F_{i}, u \in \Phi(X), i=1,2,3$.

Now we prove that all the compositions are trivial. 
For $f_{1} \wedge f_{2}$, let $f=x_{i} P\left(x_{j}\right)-\left\{x_{i} \prec x_{j}\right\}, g=P\left(x_{j}\right) x_{k}-\left\{x_{j} \succ x_{k}\right\}, i, j, k \in I$. Then $w=x_{i} P\left(x_{j}\right) x_{k}$ and

$$
\begin{aligned}
(f, g)_{w} & =x_{i} P\left(x_{j}\right) x_{k}-\left\{x_{i} \prec x_{j}\right\} x_{k}-\left(x_{i} P\left(x_{j}\right) x_{k}-x_{i}\left\{x_{j} \succ x_{k}\right\}\right) \\
& =x_{i}\left\{x_{j} \succ x_{k}\right\}-\left\{x_{i} \prec x_{j}\right\} x_{k} \\
& \equiv 0 \bmod \left(F_{3}, w\right) .
\end{aligned}
$$

For $f_{2} \wedge f_{1}$, let $f=P\left(x_{i}\right) x_{j}-\left\{x_{i} \succ x_{j}\right\}, g=x_{j} P\left(x_{k}\right)-\left\{x_{j} \prec x_{k}\right\}, i, j, k \in I$. Then $w=P\left(x_{i}\right) x_{j} P\left(x_{k}\right)$ and by equation (1.1),

$$
\begin{aligned}
(f, g)_{w} & =P\left(x_{i}\right) x_{j} P\left(x_{k}\right)-\left\{x_{i} \succ x_{j}\right\} P\left(x_{k}\right)-P\left(x_{i}\right)\left(x_{j} P\left(x_{k}\right)-\left\{x_{j} \prec x_{k}\right\}\right) \\
& =P\left(x_{i}\right)\left\{x_{j} \prec x_{k}\right\}-\left\{x_{i} \succ x_{j}\right\} P\left(x_{k}\right) \\
& \equiv\left\{x_{i} \succ\left\{x_{j} \prec x_{k}\right\}\right\}-\left\{\left\{x_{i} \succ x_{j}\right\} \prec x_{k}\right\} \\
& \equiv 0 \bmod (S, w) .
\end{aligned}
$$

For $f_{3} \wedge f_{1}$, let $f=u_{0} P\left(v_{1}\right) u_{1} \cdots P\left(v_{n}\right) u_{n} x_{i}, g=x_{i} P\left(x_{j}\right)-\left\{x_{i} \prec x_{j}\right\}, u_{0}, u_{n} \in$ $X^{*}, u_{k} \in X^{*} \backslash\{1\}, v_{l} \in \Phi_{1}(X) \backslash X, i, j \in I, n \geq 0,1 \leq k<n, 1 \leq l \leq n$, and $\left|u_{0}\right| \geq 1$ if $n=0$. Then $w=u_{0} P\left(v_{1}\right) u_{1} \cdots P\left(v_{n}\right) u_{n} x_{i} P\left(x_{j}\right)$ and

$$
\begin{aligned}
(f, g)_{w} & =u_{0} P\left(v_{1}\right) u_{1} \cdots P\left(v_{n}\right) u_{n}\left\{x_{i} \prec x_{j}\right\} \\
& \equiv 0 \bmod (S, w) .
\end{aligned}
$$

For $f_{2} \wedge f_{3}$, the proof is similar to $f_{3} \wedge f_{1}$.

For $f_{3} \wedge f_{3}^{\prime}$, we have $(f, g)_{w}=0$.

Now, we check the compositions of left and right multiplication. We prove only the cases of $f_{1} P(u)$ and $P(u) f_{3}$, where $f_{1} \in F_{1}, f_{3} \in F_{3}, u \in \Phi(X)$. Others can be similarly proved.

We may assume that $u \in \Phi_{1}(X)$.

For $f_{1} P(u)$, let $f=x_{i} P\left(x_{j}\right)-\left\{x_{i} \prec x_{j}\right\}, i, j \in I$ and $w=\overline{f P(u)}$. There are two cases to consider.

(1) $u=x_{k} \in X$. Then by using the equation (1.1),

$$
\begin{aligned}
f P(u) & =x_{i} P\left(x_{j}\right) P\left(x_{k}\right)-\left\{x_{i} \prec x_{j}\right\} P\left(x_{k}\right) \\
& =x_{i} P\left(P\left(x_{j}\right) x_{k}\right)+x_{i} P\left(x_{j} P\left(x_{k}\right)\right)-\left\{x_{i} \prec x_{j}\right\} P\left(x_{k}\right) \\
& \equiv x_{i} P\left(\left\{x_{j} \succ x_{k}\right\}\right)+x_{i} P\left(\left\{x_{j} \prec x_{k}\right\}\right)-\left\{\left\{x_{i} \prec x_{j}\right\} \prec x_{k}\right\} \\
& \equiv\left\{x_{i} \prec\left\{x_{j} \succ x_{k}\right\}\right\}+\left\{x_{i} \prec\left\{x_{j} \prec x_{k}\right\}\right\}-\left\{\left\{x_{i} \prec x_{j}\right\} \prec x_{k}\right\} \\
& \equiv 0 \bmod (S, w) .
\end{aligned}
$$

(2) $u \in \Phi_{1}(X) \backslash X$. Then

$$
\begin{aligned}
f P(u) & =x_{i} P\left(x_{j}\right) P(u)-\left\{x_{i} \prec x_{j}\right\} P(u) \\
& =x_{i} P\left(P\left(x_{j}\right) u\right)+x_{i} P\left(x_{j} P(u)\right)-\left\{x_{i} \prec x_{j}\right\} P(u) .
\end{aligned}
$$

By Lemma 3.2 we have $P\left(x_{j}\right) u \equiv \sum \alpha_{l} u_{l} \bmod \left(F_{1} \cup F_{2}, \overline{\left.P\left(x_{j}\right) u\right)}\right.$, where $u_{l} \in \Phi_{1}(X) \backslash X$. Then

$$
\begin{aligned}
f P(u) & \equiv x_{i} P\left(\sum \alpha_{l} u_{l}\right)+x_{i} P\left(x_{j} P(u)\right)-\left\{x_{i} \prec x_{j}\right\} P(u) \\
& \equiv \sum \alpha_{l} x_{i} P\left(u_{l}\right)+x_{i} P\left(x_{j} P(u)\right)-\left\{x_{i} \prec x_{j}\right\} P(u) \\
& \equiv 0 \bmod (S, w) .
\end{aligned}
$$


For $P(u) f_{3}$, let $f=P\left(v_{1}\right) u_{1} \cdots P\left(v_{n}\right) u_{n}$, where $u_{n} \in X^{*}, u_{t} \in X^{*} \backslash\{1\}, v_{l} \in$ $\Phi_{1}(X) \backslash X, n \geq 1,1 \leq t<n, 1 \leq l \leq n$, and let $w=\overline{P(u) f}$. Then

$$
P(u) f=P(u) P\left(v_{1}\right) u_{1} \cdots P\left(v_{n}\right) u_{n} .
$$

By Lemma 3.2. we have $P(u) P\left(v_{1}\right) \equiv \sum \alpha_{i} P\left(w_{i}\right) \bmod \left(F_{1} \cup F_{2}, \overline{P(u) P\left(v_{1}\right)}\right)$, where each $w_{i} \in \Phi_{1}(X) \backslash X$. Then

$$
\begin{aligned}
P(u) f & \equiv \sum \alpha_{i} P\left(w_{i}\right) u_{1} \cdots P\left(v_{n}\right) u_{n} \\
& \equiv 0 \bmod (S, w) .
\end{aligned}
$$

So, all compositions in $S$ are trivial. The proof is complete.

We now prove Theorem 1.1 .

The proof of Theorem 1.1. Let $R=F_{1} \cup F_{2}$. Then for any $u \notin \operatorname{Irr}\left(R^{\operatorname{comp}}\right)$, we have $u=\left.v\right|_{\bar{r}}$, where $r \in R^{c o m p},\left.v\right|_{r}$ is a normal $R^{c o m p}$-word. Then $f=\left.v\right|_{r} \in$ $\operatorname{Id}\left(R^{c o m p}\right)=I d(R) \subseteq I d(S)$. Since $S$ is a Gröbner-Shirshov basis in $R B(X)$, by Theorem 2.1. we have $\bar{f}=\left.w\right|_{\bar{s}}$ for some $w \in \Phi^{\star}(X), s \in S$. That is, $u=\left.v\right|_{\bar{r}}=$ $\bar{f} \notin \operatorname{Irr}(S)$. So, we have that $\operatorname{Irr}\left(R^{\operatorname{comp}}\right) \supset \operatorname{Irr}(S) \supset X$. Since $\operatorname{Irr}\left(R^{\mathrm{comp}}\right)$ is an $F$-basis of $U(D), D$ can be embedded into $U(D)$.

\section{ACKNOWLEDGEMENTS}

The authors would like to thank Professor L. A. Bokut for his guidance, useful discussions and enthusiastic encouragement. The authors thank Professor L. Guo for his valuable suggestions on this paper. The authors also thank the referee for some valuable comments on this paper.

\section{REFERENCES}

1. G. E. Andrew, L. Guo, W. Keigher, K. Ono, Baxter algebras and Hopf algebras, Trans. Amer. Math. Soc. 355 (2003), 4639-4656. MR1990765 (2004f:16061)

2. M. Aymon and P.-P. Grivel, Un theoreme de Poincaré-Birkhoff-Witt pour les algebres de Leibniz, Comm. Algebra 31 (2003), 527-544. MR1968912 (2004b:17004)

3. G. Baxter, An analytic problem whose solution follows from a simple algebraic identity, Pacific J. Math. 10 (1960), 731-742. MR0119224(22:9990)

4. L. A. Bokut, Yuqun Chen and Xueming Deng, Gröbner-Shirshov bases for Rota-Baxter algebras, Siberian Math. J. 51(6) (2010), 978-988.

5. P. Cartier, On the structure of free Baxter algebras, Adv. Math. 9 (1972), 253-265. MR0338040 (49:2807)

6. K. Ebrahimi-Fard, Loday-type algebras and the Rota-Baxter relation, Lett. Math. Phys. 61(2) (2002), 139-147. MR.1936573 (2004b:17003)

7. K. Ebrahimi-Fard and L. Guo, Rota-Baxter algebras and dendriform algebras, Journal of Pure and Applied Algebra 212(2) (2008), 320-339. MR2357335 (2008i:16036)

8. K. Ebrahimi-Fard and L. Guo, Free Rota-Baxter algebras and rooted trees, J. Algebra and Its Applications 7 (2008), 167-194. MR2417040 (2009b:05260)

9. P. S. Kolesnikov, Conformal representations of Leibniz algebras, Siberian Math. J. 49 (2008), 429-435. MR2442537(2009e:17005)

10. J.-L. Loday, Dialgebras, in Dialgebras and related operads, Lecture Notes in Math., 1763, Springer, Berlin, 2001, 7-66. MR.1860994(2002i:17004)

11. J.-L. Loday, Scindement d'associativité et algèbres de Hopf. Actes des Journées Mathématiques à la Mémoire de Jean Leray, 155-172, Sémin. Congr., 9, Soc. Math. France, Paris, 2004. MR2145941 (2006a:16054) 
12. D. Quillen, Rational homotopy theory, Ann. of Math. (2) 90(2) (1969), 205-295. MR0258031 (41:2678)

13. G. Rota, Baxter operators, an introduction, in: Gian-Carlo Rota on combinatorics. Introductory papers and commentaries, Joseph P. S. Kung, editor, Birkhäuser, Boston, 1995. MR:1392961 (99b:01027)

14. I. P. Shestakov, Every Akivis algebra is linear, Geometriae Dedicata 77 (1999), 215-223. MR.1713296 (2000f:17004)

School of Mathematical Sciences, South China Normal University, Guangzhou 510631, People's Republic of China

E-mail address: yqchen@scnu.edu.cn

School of Mathematical Sciences, South China Normal University, Guangzhou 510631, People's Republic of China

E-mail address: scnuhuashimomo@126.com 